\title{
Research Article System Identification of MEMS Vibratory Gyroscope Sensor
}

\author{
Juntao Fei ${ }^{1,2}$ and Yuzheng Yang ${ }^{2}$ \\ 1 Jiangsu Key Laboratory of Power Transmission and Distribution Equipment Technology, \\ Changzhou 213022, China \\ ${ }^{2}$ College of Computer and Information, Hohai University, Changzhou 213022, China
}

Correspondence should be addressed to Juntao Fei, jtfei@yahoo.com

Received 14 May 2011; Revised 17 July 2011; Accepted 1 August 2011

Academic Editor: Massimo Scalia

Copyright (C) 2011 J. Fei and Y. Yang. This is an open access article distributed under the Creative Commons Attribution License, which permits unrestricted use, distribution, and reproduction in any medium, provided the original work is properly cited.

\begin{abstract}
Fabrication defects and perturbations affect the behavior of a vibratory MEMS gyroscope sensor, which makes it difficult to measure the rotation angular rate. This paper presents a novel adaptive approach that can identify, in an online fashion, angular rate and other system parameters. The proposed approach develops an online identifier scheme, by rewriting the dynamic model of MEMS gyroscope sensor, that can update the estimator of angular rate adaptively and converge to its true value asymptotically. The feasibility of the proposed approach is analyzed and proved by Lyapunov's direct method. Simulation results show the validity and effectiveness of the online identifier.
\end{abstract}

\section{Introduction}

Gyroscopes are commonly used sensors for measuring angular velocity in many areas of applications such as navigation, homing, and control stabilization. Vibratory gyroscopes are the devices that transfer energy from one axis to another axis through Coriolis forces. The conventional mode of operation drives one of the modes of the gyroscope into a known oscillatory motion and then detects the Coriolis acceleration coupling along the sense mode of vibration, which is orthogonal to the driven mode. The response of the sense mode of vibration provides information about the applied angular velocity. Fabrication imperfections result in some cross-stiffness and cross-damping effects that may hinder the measurement of angular velocity of MEMS gyroscope. The angular velocity measurement and minimization of the cross-coupling between two axes are challenging problems in vibrating gyroscopes.

Ioannou and Sun [1] and Tao [2] described the model reference adaptive control. Chou and Cheng [3] proposed an integral sliding surface and derived an adaptive law to estimate 
the upper bound of uncertainties. Some control algorithms have been proposed to control the MEMS gyroscope. Batur and Sreeramreddy [4] developed a sliding mode control for a MEMS gyroscope system. Leland [5] presented an adaptive force balanced controller for tuning the natural frequency of the drive axis of a vibratory gyroscope. Novel robust adaptive controllers are proposed in [6,7] to control the vibration of MEMS gyroscope. Sung et al. [8] developed a phase-domain design approach to study the mode-matched control of MEMS vibratory gyroscope. Antonello et al. [9] used extremum-seeking control to automatically match the vibration mode in MEMS vibrating gyroscopes. Feng and Fan [10] presented an adaptive estimator-based technique to estimate the angular motion by providing the Coriolis force as the input to the adaptive estimator and to improve the bandwidth of microgyroscope. Tsai and Sue [11] proposed integrated model reference adaptive control and time-varying angular rate estimation algorithm for micromachined gyroscopes. Raman et al. [12] developed a closed-loop digitally controlled MEMS gyroscope using unconstrained sigma-delta force balanced feedback control. Park et al. [13] presented an adaptive controller for a MEMS gyroscope which drives both axes of vibration and controls the entire operation of the gyroscope.

The adaptive control of MEMS gyroscope is system identification problem. The identification is a very rich investigation subject in the last decades. The persistence of excitation is the most important problem in the system identification. There are several ways to deal with it; one is to use a sufficient number of frequencies in the reference signal. Sometimes, the use of multiestimation-type schemes with appropriate switching among the various single multiestimation schemes integrating the whole tandem has been done successfully since this guarantees directly sufficiently frequency richness/excitation persistence. Guillerna et al. [14] proposed a robustly stable multiestimation scheme for adaptive control and identification with model reduction issues. Sen and Alonso [15] presented adaptive control of time-invariant systems with discrete delays subject to multiestimation. Moreover, there are some articles devoted to identification in practical real problems. Paulraj and Sumathi [16] compared the redundant constraints identification methods in linear programming problems. Ho and Chan [17] developed hybrid differential evolution algorithm for parameter estimation of differential equation models with application to HIV dynamics. Blais [18] derived a novel least squares method for practitioners.

In this paper, a novel adaptive online identifier is designed to estimate the angular rate and system parameters. The motivation of this paper is to propose a novel series-parallel online identifier that could estimate all the system parameters using observed state and control signals. The advantage of proposed adaptive approach is that it is easy to implement in practice and it avoids the complicated algorithm derivation; therefore, it is better than other control algorithm for the vibratory MEMS gyroscope.

The paper is organized as follows. In Section 2, the dynamics of MEMS gyroscope sensor is introduced. In Section 3, the online identifier is developed to achieve the system parameters and angular rate. In Section 4, simulation results are presented to verify the design of online identifier. Conclusion is provided in Section 5.

\section{Dynamics of MEMS Gyroscope}

A typical MEMS vibratory gyroscope sensor configuration includes a proof mass suspended by spring beams, electrostatic actuations, and sensing mechanisms for forcing an oscillatory motion and sensing the position and velocity of the proof mass as well as a rigid frame which 


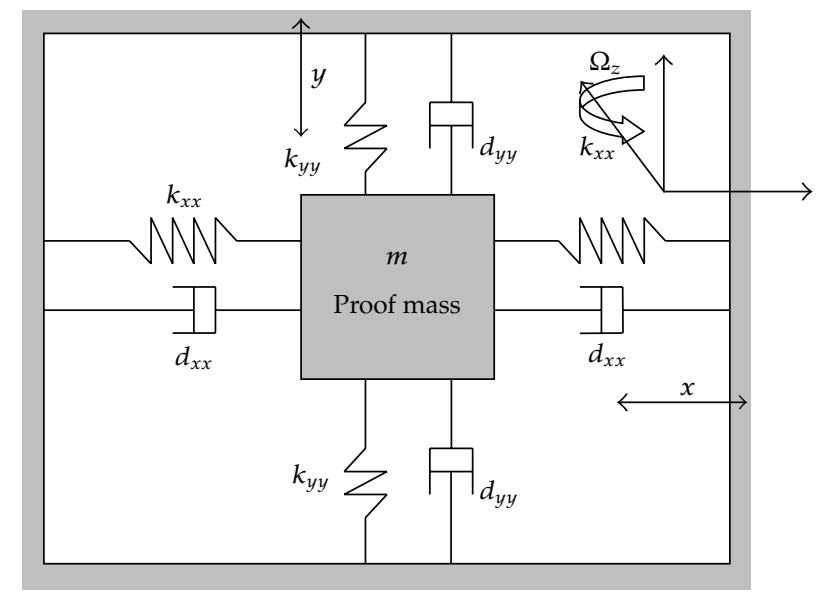

Figure 1: Simplified model of a z-axis MEMS gyroscope sensor.

is rotated along the rotation axis. Dynamics of a MEMS gyroscope sensor is derived from Newton's law in the rotating frame.

As we known, Newton's law in the rotating frame becomes

$$
F_{r}=F_{\text {phy }}+F_{\text {centri }}+F_{\text {Coriolis }}+F_{\text {Euler }}=m a_{r} .
$$

In (2.1), $F_{r}$ is the total applied force to the proof mass in the gyro frame, $F_{\text {phy }}$ is the total physical force to the proof mass in the inertial frame, $F_{\text {centri }}$ is the centrifugal force, $F_{\text {Coriolis }}$ is the Coriolis force, $F_{\text {Euler }}$ is the Euler force, and $a_{r}$ is the acceleration of the proof mass with respect to the gyro frame. $F_{\text {phy }}, F_{\text {Coriolis, }}$ and $F_{\text {Euler }}$ are inertial forces caused by the rotation of the gyro frame.

With the definition of $r_{r}, v_{r}$ as the position and velocity vectors relative to the rotating gyroscope frame and $\Omega$ as the angular velocity vector of the gyroscope frame, the expressions for the inertial forces reduce to

$$
F_{\text {Coriolis }}=-2 m \Omega \times v_{r}, \quad F_{\text {centri }}=-m \Omega \times\left(\Omega \times r_{r}\right), \quad F_{\text {Euler }}=-m \frac{d \Omega}{d t} \times r_{r},
$$

then

$$
m a_{r}+m \Omega \times\left(\Omega \times r_{r}\right)+2 m \Omega \times v_{r}+m \dot{\Omega} \times r_{r}=F_{\text {phy }}
$$

where $F_{\text {phy }}$ contains spring, damping, and control forces applied to the proof mass.

In a $z$-aixs gyroscope sensor, by supposing the stiffness of spring in $z$ direction much larger than that in $x, y$ directions, motion of poof mass is constrained to only along the $x-y$ plan as shown in Figure 1. Assuming that the measured angular velocity is almost constant 
over a long enough time interval, the equation of motion of a gyroscope sensor based on (2.3) is simplified as follows

$$
\begin{aligned}
& m \ddot{x}+d_{x} \dot{x}+\left[k_{x}-m\left(\Omega_{y}^{2}+\Omega_{z}^{2}\right)\right] x+m \Omega_{x} \Omega_{y} y=u_{x}+2 m \Omega_{z} \dot{y}, \\
& m \ddot{y}+d_{y} \dot{y}+\left[k_{y}-m\left(\Omega_{x}^{2}+\Omega_{z}^{2}\right)\right] y+m \Omega_{x} \Omega_{y} x=u_{y}-2 m \Omega_{z} \dot{x},
\end{aligned}
$$

where $x$ and $y$ are the coordinates of the proof mass with respect to the gyro frame in a Cartesian coordinate system, $d_{x, y}$ and $k_{x, y}$ are damping and spring coefficients, $\Omega_{x, y, z}$ are the angular rate components along each axis of the gyro frame, and $u_{x, y}$ are the control forces. The last two terms in (2.4), $2 m \Omega_{z} \dot{y}$ and $2 m \Omega_{z} \dot{x}$, are the Coriolis forces and are used to reconstruct the unknown input angular rate $\Omega_{z}$. Under typical assumptions $\Omega_{x} \approx \Omega_{y} \approx 0$, only the component of the angular rate $\Omega_{z}$ causes a dynamic coupling between $x$ and $y$ axes.

Taking fabrication imperfections into account, which cause extra coupling between $x$ and $y$ axes, the governing equation for a $z$-axis MEMS gyroscope sensor is

$$
\begin{aligned}
& m \ddot{x}+d_{x x} \dot{x}+d_{x y} \dot{y}+k_{x x} x+k_{x y} y=u_{x}+2 m \Omega_{z} \dot{y}, \\
& m \ddot{y}+d_{x y} \dot{x}+d_{y y} \dot{y}+k_{x y} x+k_{y y} y=u_{y}-2 m \Omega_{z} \dot{x},
\end{aligned}
$$

In (2.5), $d_{x x}$ and $d_{y y}$ are damping, $k_{x x}$ and $k_{y y}$ are spring coefficients $d_{x y}$, and $k_{x y}$, called quadrature errors, are coupled damping and spring terms, respectively, mainly due to the asymmetries in suspension structure and misalignment of sensors and actuators. The coupled spring and damping terms are unknown, but can be assumed to be small. The nominal values of the $x$ and $y$ axes spring and damping terms are known, but there are small unknown variations. The proof mass can be determined accurately.

Dividing both sides of (2.5) by $m, q_{0}$, and $w_{0}^{2}$, which are a reference mass, length, and natural resonance frequency, respectively, where $m$ is the proof mass of a gyroscope sensor, we get the form of the nondimensional equation of motion as

$$
\begin{aligned}
& \ddot{x}+d_{x x} \dot{x}+d_{x y} \dot{y}+w_{x}^{2} x+w_{x y} y=u_{x}+2 \Omega_{z} \dot{y}, \\
& \ddot{y}+d_{x y} \dot{x}+d_{y y} \dot{y}+w_{x y} x+w_{y}^{2} y=u_{y}-2 \Omega_{z} \dot{x},
\end{aligned}
$$

where $d_{x x} / m w_{0} \rightarrow d_{x x}, d_{x y} / m w_{0} \rightarrow d_{x y}, d_{y y} / m w_{0} \rightarrow d_{y x y}, \Omega_{z} / w_{0} \rightarrow \Omega_{z}, \sqrt{k_{x x} / m w_{0}^{2}} \rightarrow$ $w_{x}, \sqrt{k_{y y} / m w_{0}^{2}} \rightarrow w_{y}, k_{x y} / m w_{0}^{2} \rightarrow w_{x y}$. Rewrite the gyroscope sensor model (2.6) in statespace form as

$$
\dot{x}=\mathbf{A} x+\mathbf{B} u,
$$




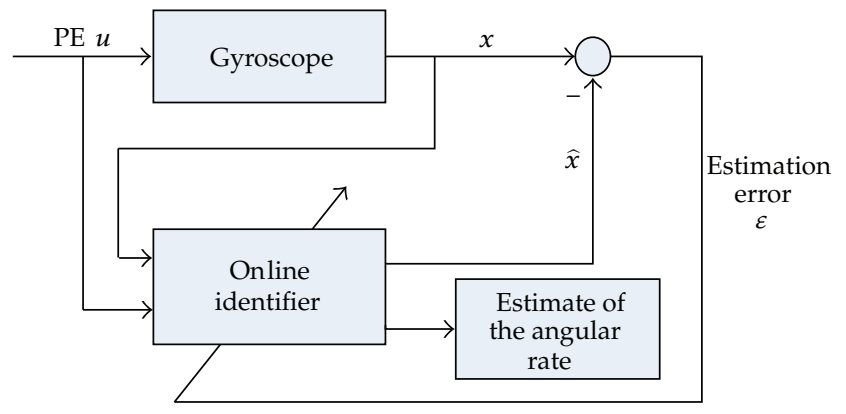

Figure 2: Block diagram of the online identifier.

where

$$
\begin{gathered}
x=\left[\begin{array}{l}
x \\
\dot{x} \\
y \\
\dot{y}
\end{array}\right], \quad \mathbf{A}=\left[\begin{array}{cccc}
0 & 1 & 0 & 0 \\
-w_{x}^{2} & -d_{x x} & -w_{x y} & -\left(d_{x y}-2 \Omega_{z}\right) \\
0 & 0 & 0 & 1 \\
-w_{x y} & -\left(d_{x y}+2 \Omega_{z}\right) & -w_{y}^{2} & -d_{y y}
\end{array}\right], \\
\mathbf{B}=\left[\begin{array}{ll}
0 & 0 \\
1 & 0 \\
0 & 0 \\
0 & 1
\end{array}\right], \quad u=\left[\begin{array}{l}
u_{x} \\
u_{y}
\end{array}\right] .
\end{gathered}
$$

From (2.8), it is obvious that all the unknown or uncertain parameters including the input angular rate are lumped in the $\mathbf{A}$. Then, this paper will develop a novel adaptive approach that can identify the system matrix $\mathbf{A}$ in an online fashion.

The dynamics of MEMS gyroscope sensor described by (2.6) can be considered as a mass, spring, and damper system, which implies that $\mathbf{A}$ is stable. With the assumption that the control inputs $u_{x}$ and $u_{y}$ are bounded, the system state vector $x$ is then also bounded. This prior knowledge will be used for the design of the online identifier.

\section{The Design of Online Identifier}

The objective of this section is to generate an adaptive law for identifying $\mathbf{A}$ online by using the observed signals $x(t)$ and $u(t)$. Figure 2 shows the block diagram of the online identifier.

First of all, rewrite the dynamic model (2.7) by adding and subtracting a term $\mathbf{A}_{m} x$, where $\mathbf{A}_{m}$ is an arbitrary stable matrix:

$$
\dot{x}=\mathbf{A}_{m} x+\left(\mathbf{A}-\mathbf{A}_{m}\right) x+\mathbf{B} u .
$$


The adaptive law for generating the estimate $\widehat{\mathbf{A}}$ of $\mathbf{A}$ is to be driven by the estimation error

$$
\varepsilon=x-\widehat{x},
$$

where $\hat{x}$ is the estimated value of $x$, that is, the state output of the identifier, by using the estimate $\widehat{\mathbf{A}}$. The state $\widehat{x}$ is generated by an equation that has the same form as the gyroscope sensor model but with A replaced by $\widehat{\mathbf{A}}$. Deriving from the gyroscope sensor equation (3.1), the model of the online identifier is

$$
\dot{\hat{x}}=\mathbf{A}_{m} \widehat{x}+\left(\widehat{\mathbf{A}}-\mathbf{A}_{m}\right) x+\mathbf{B} u
$$

The estimation error $\varepsilon=x-\widehat{x}$ satisfies the differential equation

$$
\dot{\varepsilon}=\mathbf{A}_{m} \varepsilon-\tilde{\mathbf{A}} x,
$$

where

$$
\widetilde{\mathbf{A}}=\widehat{\mathbf{A}}-\mathbf{A} .
$$

Equation (3.4) indicates how the parameter error affects the estimation errors $\varepsilon$. Because $\mathbf{A}_{m}$ is stable, zero parameter error implies that $\varepsilon$ converges to zero exponentially. Indeed, $\tilde{\mathbf{A}}$ is unknown and $\varepsilon$ is the only measured signal that we can monitor in practice to check the successfulness of estimation. We have the following theorem to gain the objective.

Theorem 3.1. Consider the online identifier system (3.3) and with the control input u. By utilizing parameter adjusting law (3.6)

$$
\dot{\widetilde{\mathbf{A}}}=\dot{\widehat{\mathbf{A}}}=\mathbf{P} \varepsilon x^{T},
$$

then the proposed adaptive identifier scheme can guarantee the following properties:

(1) the identifier scheme is stable;

(2) $\lim _{t \rightarrow \infty} \varepsilon(t)=\lim _{t \rightarrow \infty}(x-\widehat{x})=0$, namely, asymptotic observer property;

(3) $\lim _{t \rightarrow \infty} \dot{\widehat{\mathbf{A}}}(t)=\lim _{t \rightarrow \infty} \dot{\tilde{\mathbf{A}}}(t)=0$, namely, asymptotic preidentification property;

(4) $\lim _{t \rightarrow \infty} \widehat{\mathbf{A}}(t)=\mathbf{A}, \lim _{t \rightarrow \infty} \tilde{\mathbf{A}}(t)=0$, namely, asymptotic identification property if the persistent excitation condition is satisfied.

Proof. Utilizing the measured signals, we assume the adaptive law is of the form

$$
\dot{\hat{\mathbf{A}}}=\mathbf{F}(\varepsilon, x, \widehat{x}, u),
$$

where $\mathbf{F}$ is the function of measured signals, and is to be chosen so that the equilibrium state

$$
\widehat{\mathbf{A}}_{e}=\mathbf{A}, \quad \varepsilon_{e}=0
$$


of the differential equation described by (3.4) and (3.7) is uniformly stable, or if possible, uniformly asymptotically stable, or, even better, exponentially stable. (3.4):

Consider the following Lyapunov function candidate of the estimation error system

$$
V(\varepsilon, \tilde{\mathbf{A}})=\varepsilon^{T} \mathbf{P} \varepsilon+\operatorname{tr}\left(\tilde{\mathbf{A}}^{T} \tilde{\mathbf{A}}\right)
$$

where $\operatorname{tr}(\mathbf{A})$ denotes the trace of the matrix $\mathbf{A}$ and $\mathbf{P}=\mathbf{P}^{T}>\mathbf{0}$ is chosen as the solution of the Lyapunov equation:

$$
\mathbf{A}_{m}^{T} \mathbf{P}+\mathbf{P} \mathbf{A}_{m}=-\mathbf{Q}
$$

where $\mathbf{Q}=\mathbf{Q}^{T}>\mathbf{0}$, whose existence is guaranteed by the stability of $\mathbf{A}_{m}$.

The time derivative $\dot{V}$ of $V$ along the trajectory of (3.4), (3.7) is

$$
\begin{aligned}
\dot{V} & =\varepsilon^{T} \mathbf{P} \dot{\varepsilon}+\dot{\varepsilon}^{T} \mathbf{P} \varepsilon+\operatorname{tr}\left(\dot{\tilde{\mathbf{A}}}^{T} \tilde{\mathbf{A}}+\widetilde{\mathbf{A}}^{T} \dot{\tilde{\mathbf{A}}}\right) \\
& =\varepsilon^{T}\left(\mathbf{A}_{m}^{T} \mathbf{P}+\mathbf{P} \mathbf{A}_{m}\right) \varepsilon-2 \varepsilon^{T} \mathbf{P} \tilde{\mathbf{A}} x+\operatorname{tr}\left(\dot{\tilde{\mathbf{A}}}^{T} \tilde{\mathbf{A}}+\tilde{\mathbf{A}}^{T} \dot{\tilde{\mathbf{A}}}\right) .
\end{aligned}
$$

Use the properties of trace of a matrix

$$
\dot{V}=-\varepsilon^{T} \mathbf{Q} \varepsilon+2 \operatorname{tr}\left(\dot{\tilde{\mathbf{A}}} \tilde{\mathbf{A}}^{T}-\mathbf{P} \varepsilon \mathbf{x}^{T} \tilde{\mathbf{A}}^{T}\right)
$$

The obvious choice for $\dot{\widetilde{A}}$ to make $\dot{V}$ negative is

$$
\dot{\widetilde{\mathbf{A}}}=\dot{\widehat{\mathbf{A}}}=F=\mathbf{P} \varepsilon x^{T}
$$

This adaptive law yields

$$
\dot{V}=-\varepsilon^{T} \mathbf{Q} \varepsilon \leq 0 .
$$

Equation (3.14) implies that the equilibrium $\widehat{\mathbf{A}}_{e}=\mathbf{A}, \varepsilon_{e}=0$ of the respective equations is uniformly stable and $\widetilde{\mathbf{A}}, \varepsilon$ are all uniformly bounded for all $t$; that is, the online identifier scheme is stable. On condition that $x$ is bounded, $\dot{\varepsilon}$ is also bounded obtained from (3.4).

It can be proved that $\lim _{t \rightarrow \infty} \varepsilon(t)=\lim _{t \rightarrow \infty}(x-\hat{x})=0, \lim _{t \rightarrow \infty} \dot{\widehat{\mathbf{A}}}(t)=\lim _{t \rightarrow \infty} \dot{\widetilde{\mathbf{A}}}(t)=0$ using the following Barbalat's lemma.

Lemma 3.2. If $f(t)$ is a uniformly continuous function, such that $\lim _{t \rightarrow \infty} \int_{0}^{t} f(\tau) d \tau$ exists and is finite, then $f(t) \rightarrow 0$ as $t \rightarrow \infty$.

Corollary 3.3. If $g, \dot{g} \in L_{\infty}$ and $g \in L_{p}$, for some $p \in[1, \infty)$, then $g(t) \rightarrow 0$ as $t \rightarrow \infty$. 
Since

$$
\dot{V}=-\varepsilon^{T} \mathbf{Q} \varepsilon \leq-\lambda_{\min }(\mathbf{Q})|\varepsilon|^{2} \leq 0,
$$

where $\lambda_{\min }(\mathbf{Q})$ is the minimum eigenvalue of $\mathbf{Q}$ and satisfies $\lambda_{\min }>0$, inequality (3.15) implies that $\varepsilon$ is integrable as $\int_{0}^{t}|\varepsilon|^{2} d t \leq\left(1 / \lambda_{\min }\right)[V(0)-V(t)]$. Since $V(0)$ is bounded and $0 \leq V(t) \leq V(0)$, it can be concluded that $\lim _{t \rightarrow \infty} \int_{0}^{t}|\varepsilon|^{2} d t$ is bounded. Since $\lim _{t \rightarrow \infty} \int_{0}^{t}|\varepsilon|^{2} d t$, $\varepsilon$ and $\dot{\varepsilon}$ are bounded, according to Barbalat's lemma, $\lim _{t \rightarrow \infty} \varepsilon(t)=0$, which, in turn, implies that $\|\dot{\hat{\mathbf{A}}}\| \rightarrow \mathbf{0}$. Therefore, it can be concluded that this scheme is not only an asymptotic identifier but also an asymptotic observer.

Like in other adaptive control problems, the persistent excitation condition is an important factor to estimate the angular rate correctly. From (2.7), (2.8), the dynamics of a MEMS gyroscope sensor can be considered as a fourth-order system, which implies that if the control input $u$ contains two different nonzero frequencies, then the persistency of excitation is satisfied. Then, we define

$$
u_{x}=A_{1} \sin \left(w_{1} t\right), \quad u_{y}=A_{2} \sin \left(w_{2} t\right)
$$

where $w_{1}, w_{2}$ satisfy $w_{1} \neq w_{2}, w_{1} \neq 0, w_{2} \neq 0$. Under these assumptions, the estimate $\widehat{\mathbf{A}}$ converges to its true value $\mathbf{A}$, namely, $\lim _{t \rightarrow \infty} \widehat{\mathbf{A}}(t)=\mathbf{A}, \lim _{t \rightarrow \infty} \tilde{\mathbf{A}}(t)=0$.

In summary, if $u_{x}=A_{1} \sin \left(w_{1} t\right)$ and $u_{y}=A_{2} \sin \left(w_{2} t\right)$ are used, then $\varepsilon$ converge to zero asymptotically. Consequently, angular rate and system parameters converge to their true values.

This completes the proof of the theorem.

Remark 3.4. In this section, we consider the design of online parameter estimators for the plant that is stable, whose states are accessible for measurement and whose input $u$ is bounded. Because no feedback is used and the plant is not disturbed by any signal other than $u$, the stability of the plant is not an issue. The main concern, therefore, is the stability properties of the estimator or adaptive law that generates the online identifier for the unknown plant parameters. However, it should be recognized that, in more general cases where $A$ is either unstable or critically stable, the control should be designed in a closed-loop fashion to achieve closed-loop stability.

Remark 3.5. we are able to design online parameter estimation schemes that guarantee that the estimation error $\varepsilon$ converges to zero as $t \rightarrow \infty$, that is, the predicted state $\widehat{x}$ approaches that of the plant as $t \rightarrow \infty$ and the estimated parameters change more and more slowly as time increases. Because the input signal $u$ is sufficiently rich, it is sufficient to establish parameter convergence to the true parameter values. To be sufficiently rich, $u$ has to have enough frequencies to excite all the modes of the plant.

Remark 3.6. The properties of the adaptive scheme developed in this section rely on the stability of the plant (MEMS gyroscope model) and the boundedness of the plant input $u$. Consequently, they may not be appropriate for use in connection with control problems where $u$ is the result of feedback and is, therefore, no longer guaranteed to be bounded a priori. Fortunately, online parameter estimation scheme that uses the adaptive laws with normalization does not rely on the stability of the plant and the boundedness of the plant 
input. In plain terms the unboundedness obstacle can be avoided by dividing $u$ and $x$ (plant states) with some normalizing signal $m>0$, to obtain $u / m, x / m$ belonging to $L_{\infty}$ space and use the normalized signal to drive the adaptive law instead of $u$ and $x$. In this paper, this is not discussed in detail.

\section{Simulation Study}

In this section, we will evaluate the proposed adaptive approach on the lumped MEMS gyroscope sensor model by using MATLAB/SIMULINK. The objective of the adaptive approach is to identify the parameters including the input angular velocity correctly. Parameters of the MEMS gyroscope sensor are as follows [4]:

$$
\begin{aligned}
m & =1.8 \times 10^{-7} \mathrm{~kg}, \quad k_{x x}=63.955 \frac{N}{m}, \quad k_{y y}=95.92 \frac{N}{m}, \quad k_{x y}=12.779 \frac{N}{m}, \\
d_{x x} & =1.8 \times 10^{-6} \frac{N s}{m}, \quad d_{y y}=1.8 \times 10^{-6} \frac{N s}{m}, \quad d_{x y}=3.6 \times 10^{-7} \frac{N s}{m} .
\end{aligned}
$$

Since the general displacement range of the MEMS gyroscope sensor in each axis is submicrometer level, it is reasonable to choose $1 \mu \mathrm{m}$ as the reference length $q_{0}$. Given that the usual natural frequency of each of the axles of a vibratory MEMS gyroscope sensor is in the $\mathrm{KHz}$ range, choose the $w_{0}$ as $1 \mathrm{KHz}$. The unknown angular velocity is assumed $\Omega_{z}=$ $100 \mathrm{rad} / \mathrm{s}$. Then, the nondimensional values of the MEMS gyroscope sensor parameters are as follows: $w_{x}^{2}=355.3, w_{y}^{2}=532.9, w_{x y}=70.99, d_{x x}=0.01, d_{y y}=0.01, d_{x y}=0.002$, and $\Omega_{z}=0.1$. According to (2.8), the system matrix $\mathbf{A}$ in this simulation example is then given by

$$
\mathbf{A}=\left[\begin{array}{cccc}
0 & 1 & 0 & 0 \\
-355.3 & -0.01 & -70.99 & 0.198 \\
0 & 0 & 0 & 1 \\
-70.99 & -0.202 & -532.9 & -0.01
\end{array}\right]
$$

The four eigenvalues of $\mathbf{A}$ are $-0.0044+18.2 i,-0.0044-18.2 i,-0.0056+23.62 i$, and $-0.0056-$ $23.62 i$, which verify the stability of $\mathbf{A}$. The initial value of estimator $\widehat{\mathbf{A}}$ is $\widehat{\mathbf{A}}(0)=0.9 * \mathbf{A} . x(0)$ and $\widehat{x}(0)$ are zero initial conditions.

Given the arbitrariness of stable matrix $\mathbf{A}_{m}$, we choose $\mathbf{A}_{m}=-20 * \mathbf{I}$, for the simplicity to choose the gain matrix $\mathbf{P}$. With $\mathbf{A}_{m}=-20 * \mathbf{I}, \mathbf{P}$ can be an arbitrary positive symmetrical matrix to ensure the positivity of matrix $\mathbf{Q}$. Note that the choosing of $\mathbf{P}$ should take into account the balance of the elements in $\dot{\hat{\mathbf{A}}}$. The control input forces are $u_{x}=400 \sin (2 t)$, and $u_{y}=400 \sin (10 t)$, containing two different nozero frequencies, which are sufficiently rich. The simulation results are shown in Figures 3, 4, and 5.

Figure 3 depicts the estimation errors. It is observed that the estimation errors converge to zero quickly, under the sinusoidal input of two different nonzero frequencies, which validates that the estimated states $\widehat{x}(t)$ converge to the actual states $x(t)$ asymptotically and equilibrium $\varepsilon_{e}=0$ is uniformly asymptotically stable. Error (1), Error (2), Error (3), and Errror (4) stand for $x$-axis position estimation error, $x$-axis velocity estimation error, $y$-axis position estimation error, and $y$-axis velocity estimation error, respectively. Figure 4 


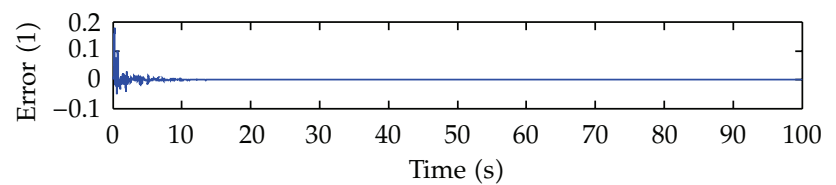

(a)

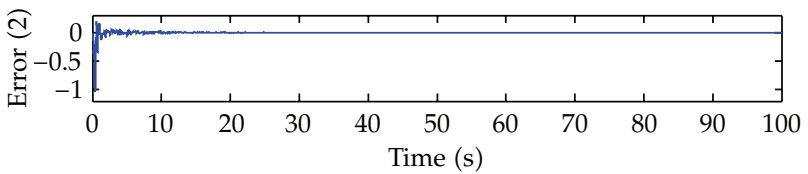

(b)

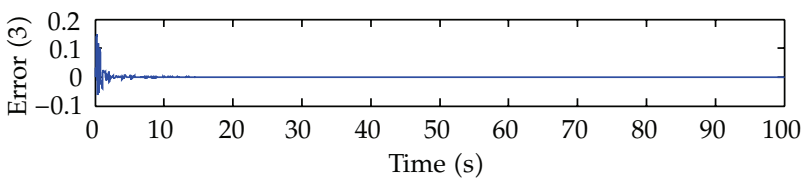

(c)

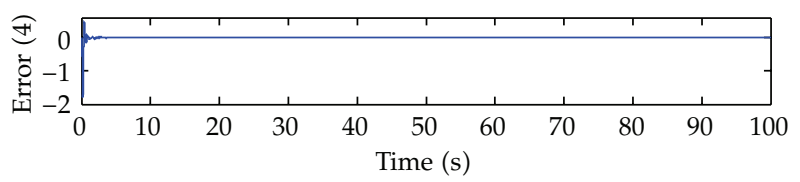

(d)

Figure 3: The estimation errors.

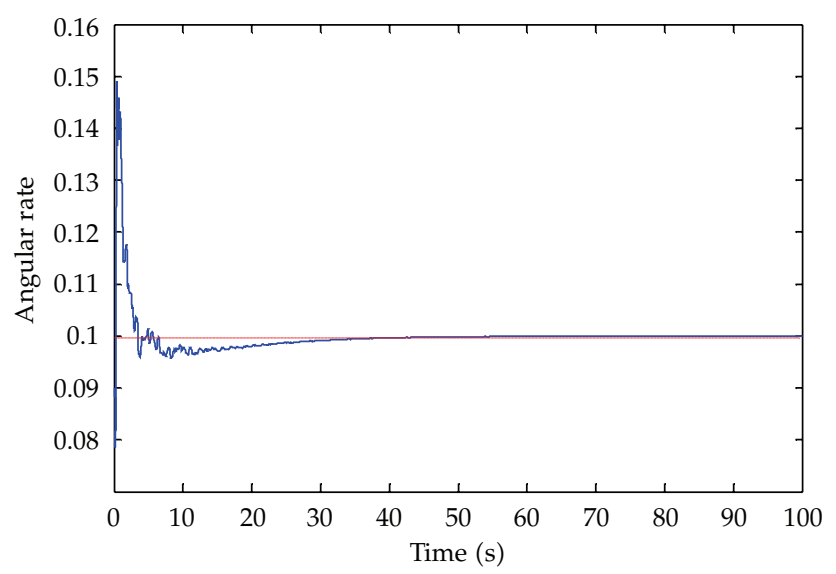

Figure 4: Adaptation of angular velocity.

obviously shows that the estimation of the angular rate reaches its actual value in finite time. The regulating time is about 10 seconds, and the overshot is approximately $49 \%$. Simulation experiments show that the regulating time and the overshot of the angular rate estimation are a pair of contradiction. Choosing a reasonable matrix $\mathbf{P}$ can find a compromise between them. Figure 5 shows that all of the estimates of $w_{x}^{2}, w_{y}^{2}, w_{x y}$ can reach their true values in a very short time, respectively, with very small overshot. 


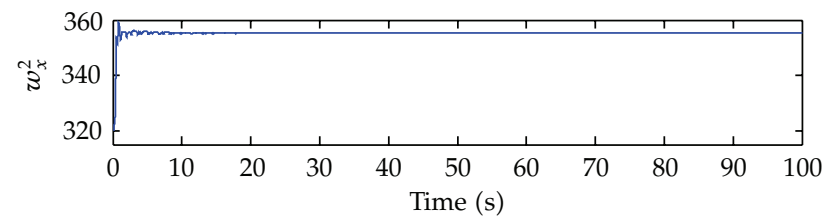

(a)

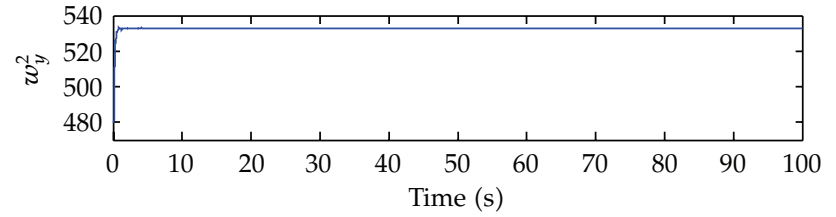

(b)

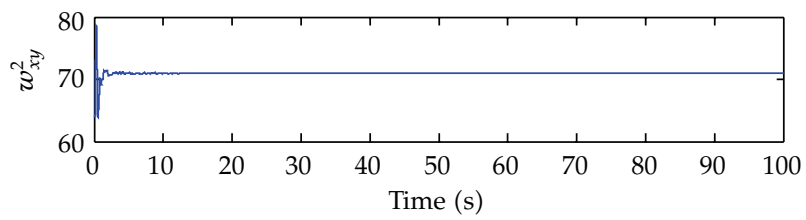

(c)

Figure 5: Adaptation of $w_{x}^{2}, w_{y}^{2}, w_{x y}$.

Simulation results verify that with the online identifier model (3.3) and the parameter adaptive law (3.12), if the persistent excitation condition is satisfied, that is, two different nonzero frequencies inputs, then the estimation errors converge to zero asymptotically and all unknown parameters, including the angular velocity go to their true values quickly without large overshot.

\section{Conclusion}

This paper investigates the design of adaptive control for MEMS gyroscope sensor. The dynamics model of the MEMS gyroscope sensor is developed and nondimensionalized. Novel adaptive approach with online identifier is proposed, and stability condition is established. Simulation results demonstrate the effectiveness of the proposed adaptive online identifier in identifying the gyroscope sensor parameters and angular rate. We should recognize that model uncertainties and external disturbances have not been considered in the proposed online adaptive identifier yet, which should be compensated for in the real application; the next step is to incorporate the term of model uncertainties and external disturbances into the online identifier to improve the robustness of the proposed method.

\section{Acknowledgments}

This work is partially supported by the National Science Foundation of China under Grant no. 61074056 and The Natural Science Foundation of Jiangsu Province under Grant no. BK2010201. One of the authors thanks the anonymous reviewer for useful comments that improved the quality of the paper. 


\section{References}

[1] P. Ioannou and J. Sun, Robust Adaptive Control, Prentice-Hall, New York, NY, USA, 1996.

[2] G. Tao, Adaptive Control Design and Analysis, John Wiley \& Sons, London, UK, 2003.

[3] C.-H. Chou and C.-C. Cheng, "A decentralized model reference adaptive variable structure controller for large-scale time-varying delay systems," IEEE Transactions on Automatic Control, vol. 48, no. 7, pp. 1213-1217, 2003.

[4] C. Batur and T. Sreeramreddy, "Sliding mode control of a simulated MEMS gyroscope," ISA Transaction, vol. 45, no. 1, pp. 99-108, 2006.

[5] R. Leland, "Adaptive control of a MEMS gyroscope using Lyapunov methods," IEEE Transactions on Control Systems Technology, vol. 14, pp. 278-283, 2006.

[6] J. Fei and C. Batur, "Robust adaptive control for a MEMS vibratory gyroscope," International Journal of Advanced Manufacturing Technology, vol. 42, no. 3-4, pp. 293-300, 2009.

[7] J. Fei and C. Batur, "A novel adaptive sliding mode control for MEMS gyroscope," ISA Transactions, vol. 48, no. 1, pp. 73-78, 2009.

[8] S. Sung, W.-T. Sung, C. Kim, S. Yun, and Y. J. Lee, "On the mode-matched control of MEMS vibratory gyroscope via phase-domain analysis and design," IEEE/ASME Transactions on Mechatronics, vol. 14, no. 4, pp. 446-455, 2009.

[9] R. Antonello, R. Oboe, L. Prandi, and F. Biganzoli, "Automatic mode matching in MEMS vibrating gyroscopes using extremum-seeking control," IEEE Transactions on Industrial Electronics, vol. 56, no. 10, pp. 3880-3891, 2009.

[10] Z. Feng and M. Fan, "Adaptive input estimation methods for improving the bandwidth of microgyroscopes," IEEE Sensors Journal, vol. 7, no. 4, pp. 562-567, 2007.

[11] N. C. Tsai and C. Y. Sue, "Integrated model reference adaptive control and time-varying angular rate estimation for micro-machined gyroscopes," International Journal of Control, vol. 83, no. 2, pp. 246-256, 2010.

[12] J. Raman, E. Cretu, P. Rombouts, and L. Weyten, “A closed-loop digitally controlled MEMS gyroscope with unconstrained sigma-delta force-feedback," IEEE Sensors Journal, vol. 83, no. 3, pp. 297-305, 2009.

[13] S. Park, R. Horowitz, S. K. Hong, and Y. Nam, "Trajectory-switching algorithm for a MEMS gyroscope," IEEE Transactions on Instrumentation and Measurement, vol. 56, no. 6, pp. 2561-2569, 2007.

[14] A. B. Guillerna, M. D. Sen, A. Ibeas, and S. A. Quesada, "Robustly stable multiestimation scheme for adaptive control and identification with model reduction issues," Discrete Dynamics in Nature and Society, no. 1, pp. 31-67, 2005.

[15] M. D. Sen and S. Alonso, "Adaptive control of time-invariant systems with discrete delays subject to multiestimation," Discrete Dynamics in Nature and Society, vol. 2006, Article ID 41973, 27 pages, 2006.

[16] S. Paulraj and P. Sumathi, "A comparative study of redundant constraint identification methods in linear programming problems," Mathematical Problems in Engineering, vol. 2010, Article ID 723402, 16 pages, 2010.

[17] W. H. Ho and A. L. F. Chan, "Hybrid taguchi-differential evolution algorithm for parameter estimation of differential equation models with application to HIV dynamics," Mathematical Problems in Engineering, vol. 2011, Article ID 514756, 14 pages, 2011.

[18] J. A. R. Blais, "Least squares for practitioners," Mathematical Problems in Engineering, vol. 2010, Article ID 508092, 19 pages, 2010. 


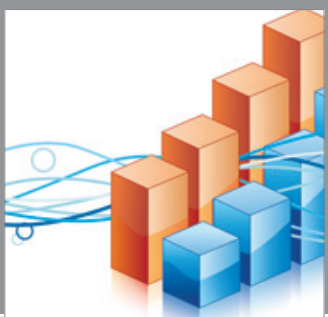

Advances in

Operations Research

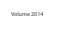

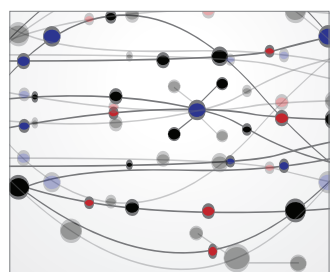

\section{The Scientific} World Journal
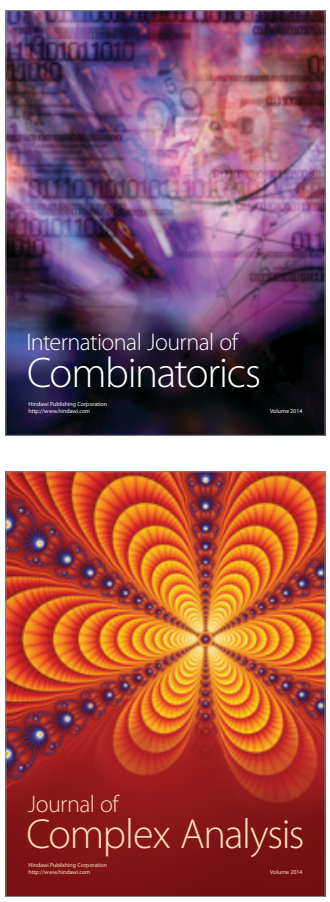

International Journal of

Mathematics and

Mathematical

Sciences
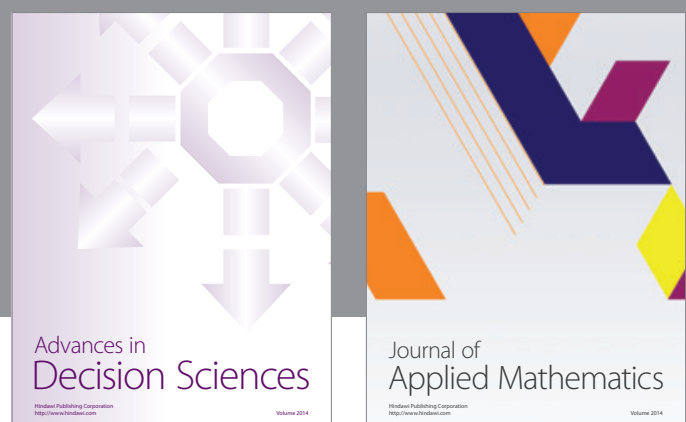

Journal of

Applied Mathematics
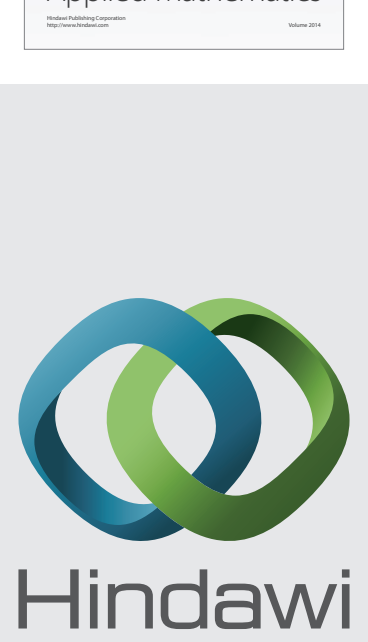

Submit your manuscripts at http://www.hindawi.com
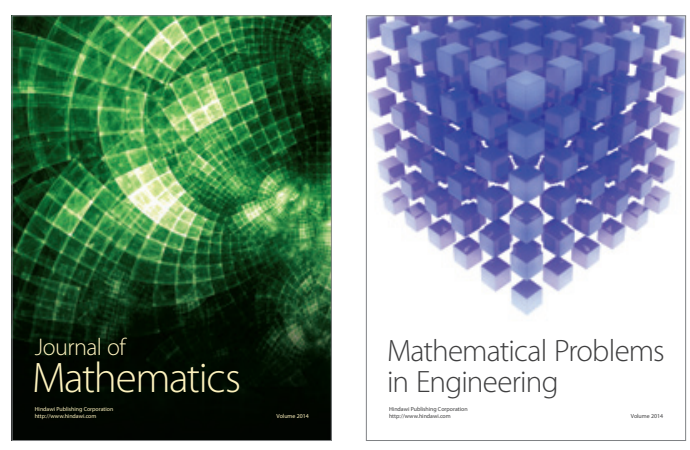

Mathematical Problems in Engineering
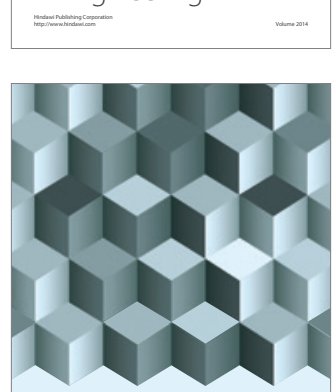

Journal of

Function Spaces
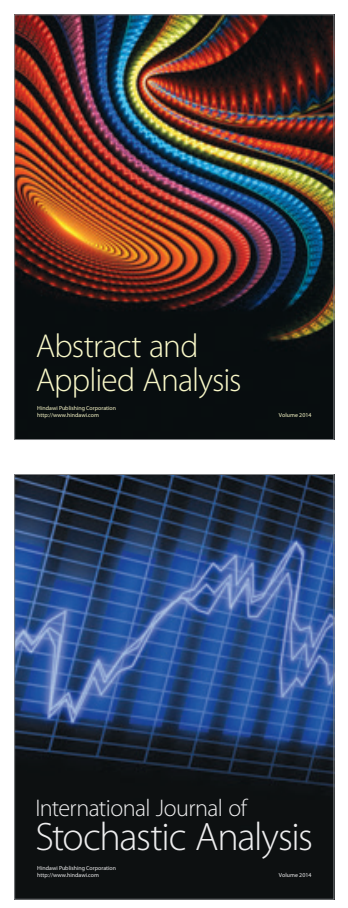

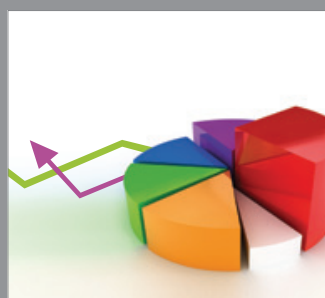

ournal of

Probability and Statistics

Promensencen
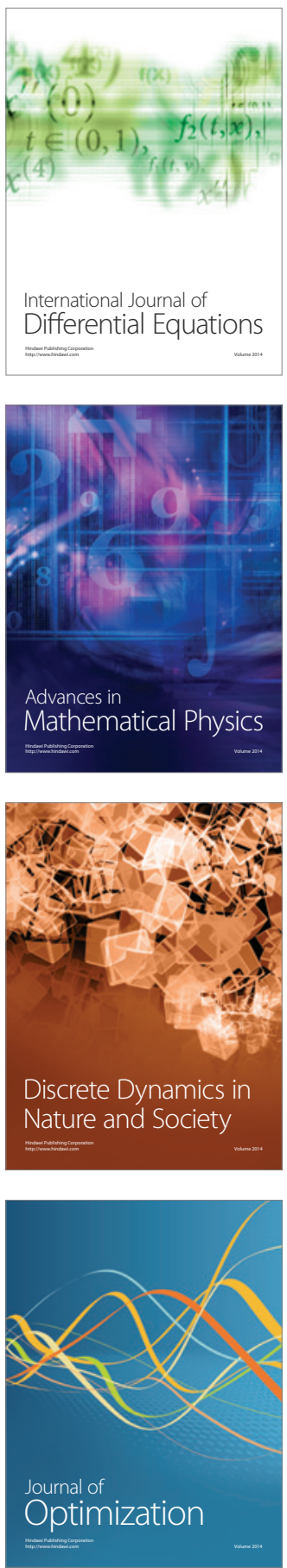